\title{
Surgical Management of Proximal Femoral Fractures by Proximal Femoral Nailing-An Institutional Experience
}

ISSN: 2576-8875

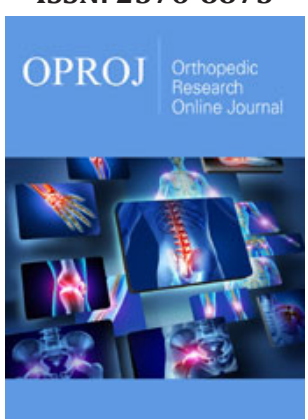

For HTML Version scan this QR code:

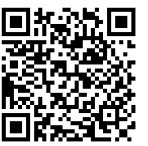

${ }^{* 1}$ Corresponding author: Madhan Jeyaraman, Junior Resident, Department of Orthopaedics,JJM Medical College, India

Submission: 些 February 18, 2019

Published: 齿 March 07, 2019

Volume 5 - Issue 2

How to cite this article: Ravinath $\mathrm{T}$, Madhan J, Kartavya C, Ravi W A, Sabarish $\mathrm{K}$, et al. Surgical Management of Proximal Femoral Fractures by Proximal Femoral Nailing-An Institutional Experience. Ortho Res Online J. 5(2). OPROJ.000610.2019. DOI: 10.31031/OPROJ.2019.05.000610

Copyright@ Madhan Jeyaraman, This article is distributed under the terms of the Creative Commons Attribution 4.0 International License, which permits unrestricted use and redistribution provided that the original author and source are credited.

\section{Ravinath TM${ }^{1}$, Madhan Jeyaraman ${ }^{1 *}$, Kartavya Chaudhari ${ }^{1}$, Ravi Weera AV$^{1}$, Sabarish $\mathbf{K}^{1}$ and Preethi Selvaraj ${ }^{2}$ \\ ${ }^{1}$ Department of Orthopaedics, JJM Medical College, Davangere, India}

${ }^{2}$ Department of Community Medicine, Shri Sathya Sai Medical College \& Research Institute, Kancheepuram, India

\begin{abstract}
Background: Proximal femoral fractures are one of the most common fractures of the hip especially in the elderly with osteoporotic bones, usually due to low-energy trauma and in young adults due to high velocity trauma. The incidence of these fractures is rising because of increasing number of senior citizens with osteoporosis. The problems associated with proximal femoral fractures are due to substantial morbidity and mortality, malunion, implant failure and associated medical conditions. Therefore, this has led to the development of intramedullary devices in the management of proximal femoral fractures.
\end{abstract}

Objective: To analyse the efficacy and functional outcome of proximal femoral nail in proximal femoral fractures.

Materials and methods: A prospective cohort study with 153 adult patients with proximal femoral fractures were treated with proximal femoral nailing in JJM Medical College, Davangere between 2013 to 2018. The fractures included in the study were trochanteric and subtrochanteric fractures were classified according to Boyd \& Griffin and Seinsheimer classification respectively. All the cases were followed at regular intervals as per our study protocol. The functional outcome was assessed with Harris Hip Score.

Results: In the present study, 88 trochanteric fractures and 65 subtrochanteric fractures underwent surgical fixation with proximal femoral nail. The functional results assessed by Harris Hip score showed excellent in 65 cases (42.48\%), good in 46 cases (30.07\%), fair in 27 cases (17.64\%) and poor in 15 cases $(9.80 \%)$. The complications occurred in the study were tracked and were treated by appropriate methods.

Conclusion: We conclude that the proximal femoral nail is a reliable implant for proximal femoral fractures, leading to high rate of bone union restoring the anatomical alignment and reduced chance of implant failure or deformities. Intramedullary fixation has biological and biomechanical advantages with decreased the morbidity and improved functional quality of life.

Keywords: Proximal femoral fractures; Proximal femoral nail; Trochanteric fractures; Subtrochanteric fractures

\section{Introduction}

Proximal femoral fractures are one of the most common fractures around the hip joint. The incidence of proximal femoral fractures occurs in bimodal distribution. The proximal femoral fractures in the young adults are due to high velocity trauma while the elderly adult sustain injury secondary to osteoporosis [1].

Proximal femoral fractures occur typically at the junction between trabecular bone and cortical bone where the mechanical stress across the junction is highest in the femur, which is responsible for their frequent comminution. The incidence of proximal femoral fractures account for $10 \%$ to $34 \%$ of all hip fractures [2]. With increasing age, there is a significant rise in pathological fractures around hip joint. Proximal femoral fractures involve both trochanteric and subtrochanteric fractures [3]. The axial loading forces through the hip joint create a large momentum, with significant lateral tensile stresses and medial compressive loads. The muscular forces at the hip create torsional effects that lead to significant rotational 
shearing forces. During normal activities of daily living, up to 6 times the body weight is transmitted across the proximal femoral region of the femur [4]. The primary goal of surgical management is to provide the anatomical restoration of the normal abductor-leverarm mechanism of the hip joint. Biomechanically, intramedullary device is a better choice of implant for fixation of proximal femoral fractures as nail provides a support to postero-medial cortex and prevents varus collapse of the fracture site [5]. The optimal positioning of surgical implants is of paramount importance for good outcome and reducing the risk of complications. The proximal femoral nail is associated with implant failure, which can be due to $\mathrm{Z}$ effect, reverse $\mathrm{Z}$ effect, screw backout, cut through of implant through bone or implant breakage [6].

\section{Objectives}

To analyse the efficacy and functional outcome of proximal femoral nail in proximal femoral fractures.

\section{Materials and Methods}

- Health care setup - Tertiary care hospital

- $\quad$ Setting - JJM Medical College, Davangere, Karnataka

- $\quad$ Duration of the study - 2013 to 2018

- $\quad$ Type of the study - Prospective cohort study

- $\quad$ Level of evidence - Level IV

- $\quad$ Sample size - 153

- $\quad$ Sampling technique - Convenient sampling

- $\quad$ Selection of cases - Out of 209 proximal femoral fracture cases, 153 cases underwent surgical management with proximal femoral nail as per our study protocol and were taken for statistical analysis

- $\quad$ Radiological assessment - Anteroposterior view of pelvis with bilateral hips and traction with $15^{\circ}$ internal rotation of affected hip radiographs were done.

- $\quad$ Functional assessment - Harris Hip score.

- Follow up - Clinical and radiological analysis at the immediate post op period and at the end of 1, 2,6,12 and 18 months.

- Implant removal were performed to patients with clinical and radiological union of fractures at an average of $26.32 \pm 3.49$ months post-surgical procedure.

\section{Fractures included in the study}

\section{Trochanteric fracture:}

Table 1: Boyd \& Griffin's classification [7].

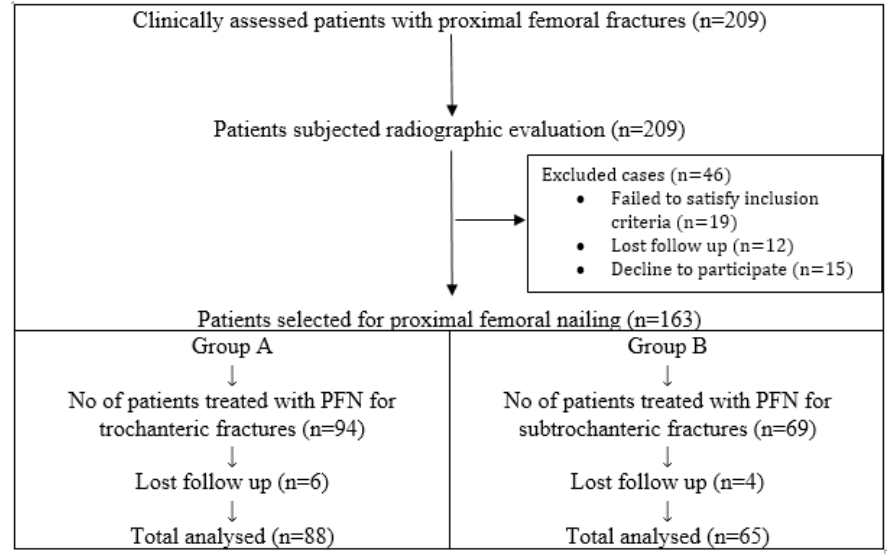

Figure 1: Patient's selection.

\section{Inclusion criteria}

- $\quad$ Patients who were in the age group from 30 - 80 years of age.

- $\quad$ Patients with clinically and radiologically diagnosed trochanteric and subtrochanteric fractures.

- $\quad$ Patients who were willing to participate in the study

\section{Exclusion criteria}

- Patients who were below 30 and above 80 years of age

- $\quad$ Patients with compound fractures, pathological fractures and fractures over ipsilateral lower limb

- $\quad$ Patients with neck of femur and shaft of femur fractures

- $\quad$ Patients who refused to participate in the study

After getting IEC and informed \& written consent from the patients and attenders, the patients enrolled in the study were subjected for thorough clinical examination. The baseline investigations and radiographic analysis such as plain X ray of the affected hip with proximal femur were analysed. The degree of osteoporosis was evaluated by Singh's index. The proximal femoral nail to be inserted were individualized. All the cases were posted for surgical management with closed or open reduction with proximal femoral nailing and followed up according to our study protocol. The other associated injuries were addressed off with appropriate management.

\begin{tabular}{|c|l|}
\hline Class & \multicolumn{1}{|c|}{ Description } \\
\hline I & Undisplaced fracture; Fracture line extends along intertrochanteric line from greater trochanter to lesser trochanter \\
\hline II & Displaced fracture; Fracture along intertrochanteric line with postero-medial commination \\
\hline III & Displaced fracture; Fracture line extending to subtrochanteric plane \\
\hline IV & Displaced fracture; Fracture occurring in atleast two planes. \\
\hline
\end{tabular}




\section{Subtrochanteric fracture:}

Table 2: Seinsheimer's classification [8].

\begin{tabular}{|c|c|c|}
\hline \multicolumn{2}{|c|}{ Class } & Description \\
\hline \multicolumn{2}{|c|}{ I } & Fracture less than $2 \mathrm{~cm}$ displacement \\
\hline \multirow{3}{*}{ II } & A & 2-part transverse fracture \\
\hline & $\mathrm{B}$ & 2-part spiral fracture with lesser trochanter attached to proximal fragment \\
\hline & $\mathrm{C}$ & 2-part spiral fracture with lesser trochanter attached to distal fragment \\
\hline \multirow{2}{*}{ III } & A & 3-part spiral fracture with lesser trochanter as separated fragment \\
\hline & $\mathrm{B}$ & 3-part spiral fracture with butterfly fragment \\
\hline \multicolumn{2}{|c|}{ IV } & Comminuted fracture with 4 or more fragments \\
\hline \multicolumn{2}{|c|}{$\mathrm{V}$} & Fracture with proximal extension into greater trochanter \\
\hline
\end{tabular}

\section{Proximal femoral nail [8-10]:}

Table 3: Long Vs Short PFN.

\begin{tabular}{|c|c|c|}
\hline Features & Long PFN & Short PFN \\
\hline Length & $340,360,380,400,420 \mathrm{~mm}$ & $180 \mathrm{~mm}$ \\
\hline $\begin{array}{c}\text { Proximal diameter } \\
\text { of nail }\end{array}$ & $17 \mathrm{~mm}$ & $15 \mathrm{~mm}$ \\
\hline $\begin{array}{c}\text { Distal diameter of nail } \\
\text { Anteversion }\end{array}$ & 10,11 and $12 \mathrm{~mm}$ & $9,10,11$ and $12 \mathrm{~mm}$ \\
\hline Medio-lateral angle & $6^{\circ}$ & $10^{\circ}$ \\
\hline Neck shaft angle & $130^{\circ}$ and $135^{\circ}$ & $6^{\circ}$ \\
\hline Proximal screws & $6.4 \mathrm{~mm}$ anti-rotation proximal stabilizing screw \& $8 \mathrm{~mm}$ distal & $\begin{array}{c}6.4 \text { mm anti-rotation proximal stabilizing screw \& } 8 \mathrm{~mm} \\
\text { distal compression screw }\end{array}$ \\
\hline Distal screws & $4.9 \mathrm{~mm}$ both static and dynamic (10mm dynamization) locking & $\begin{array}{c}4.9 \mathrm{~mm} \text { both static and dynamic (10mm dynamization) } \\
\text { locking bolts }\end{array}$ \\
\hline
\end{tabular}

\section{Advantages of proximal femoral nail}

- $\quad$ Provides more efficient load transfer

- $\quad$ Shorter lever arm can decrease tensile strain on implant and risk of implant failure

- Maintenance of controlled fracture impaction

- Acts as a buttress to prevent shaft medialisation

- Shorter operating time and lesser soft tissue dissection

- $\quad$ Proximal screws provide improved resistance to varus collapse and rotational control of head and neck fragment

- $\quad$ Allows length and rotational control in absence of lesser trochanter

- $\quad$ Presence of longitudinal slot throughout accelerates the regeneration of endosteal bone.

- $\quad$ Presence of $6^{\circ}$ medio-lateral angle helps in easy insertion of nail and a flexible distal tip to prevent stress generation and refracture.

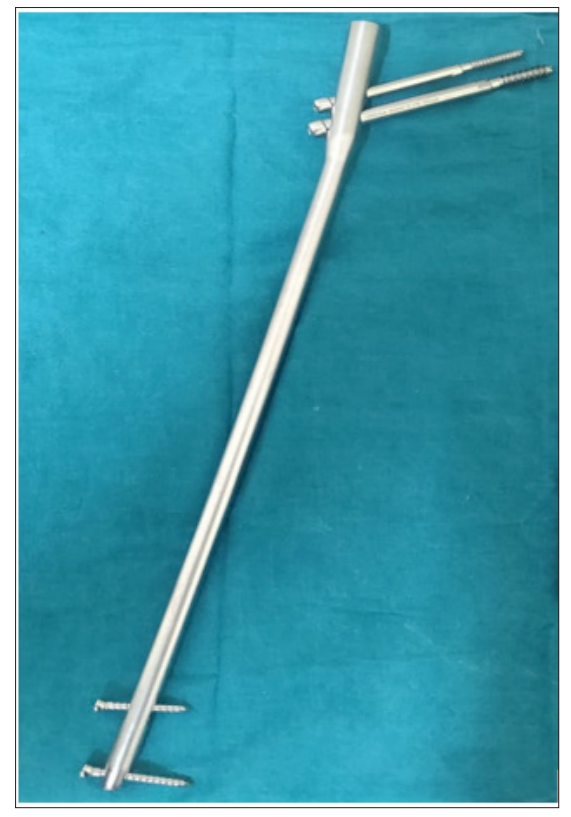

Figure 2: Long PFN. 


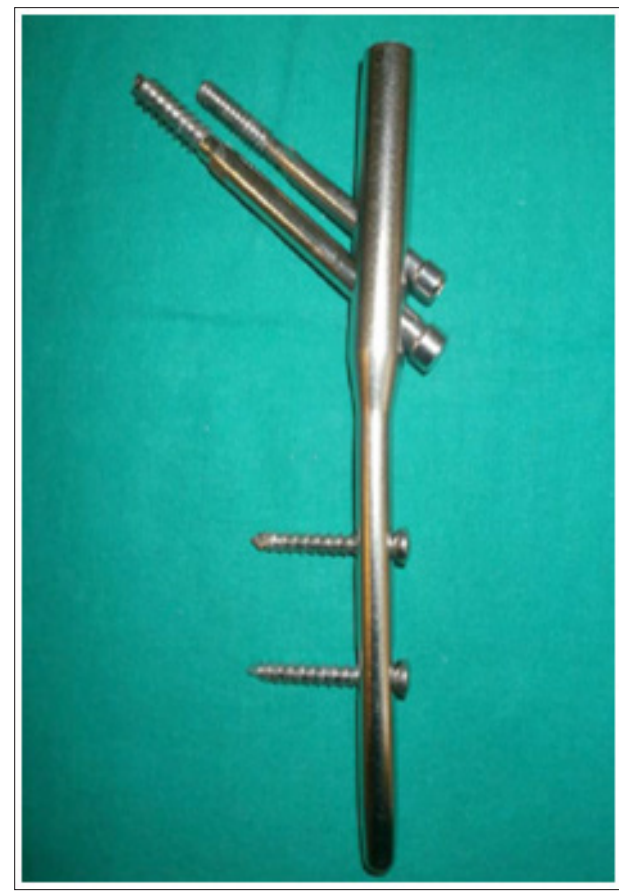

Figure 3: Short PFN.

\section{Complications of PFN [4-6,9,10]}

- Varus displacement: Associated with failure of nail fixation in proximal fragment and failure to obtain a stable reduction and internal fixation which leads to implant bending, breaking, cutting out of head and pulling of femoral shaft as described by Tyllianakis et al. [2] \& Taylor et al. [5]

- $\quad$ Z effect and Reverse Z effect: These concepts were described by Strauss et al. When superior anti-rotation screw is longer than inferior compression screw, the vertical forces increases on anti-rotation screw and start to induce cut-out produces $\mathrm{Z}$ effect or Knife effect. So that anti-rotation screw migrates medially, and the compression screw migrates laterally. The rate of occurrence of $\mathrm{Z}$ effect is $0.6-8 \%$. When the reverse of events such as migration of anti-rotation laterally and compression screw medially leads to reverse $\mathrm{Z}$ effect.

- Painful hardware: Results from backed out compression screw \& anti-rotation screw and hitching of distal tip of nail in the antero-medial cortex of femur irritating femoral musculature.

- Femoral shaft fractures: Associated with aggressive surgical insertion of nail using hammer.

- Non-union: Associated with fracture instability, loss of reduction and inadequate immobilization.

- Osteonecrosis of femoral head: Very less incidence of $0.8 \%$.

\section{Operative technique}

Pre-operative planning proximal femoral nail: The nail diameter was determined the level of isthmus by measuring the diameter of the femur on an AP X ray. The neck shaft angle was measured in unaffected side in AP X ray using goniometer.

\section{Operative technique:}

- $\quad$ Anaesthesia: All the patients were operated under either spinal anaesthesia or epidural anaesthesia.

- $\quad$ Reduction of fracture: The patients were placed in supine position on fracture table with adduction of the affected limb by $10^{\circ}-15^{\circ}$ and closed reduction of the fracture was done by the traction and internal rotation which was confirmed by the image intensifier.

- $\quad$ Preparation: The patients were prepared and draped as for any standard hip fracture fixation. Prophylactic IV antibiotic and tetanus toxoid were given in all patients $30 \mathrm{mins}$ before surgery.

- $\quad$ Approach: Make approximately $3-5 \mathrm{~cm}$ incision proximal to the tip of greater trochanter. Make a parallel incision in the fascia of gluteus Medius and split the gluteus Medius in the line with its fibres.

- $\quad$ Entry point and insertion of guide wire: Under image intensifier, the entry point was made on tip or slightly lateral to the tip of greater trochanter. In lateral view, guide wire position was confirmed in the center of the medullary cavity. The medullary canal was entered with a curved bone awl through the help of guide wire's direction.

- Reaming: Using a cannulated conical $17 \mathrm{~mm}$ reamer, proximal femur was reamed for about $7 \mathrm{cms}$. Distal femur was reamed with successive number of ordinary reamers depending on the calculated nail diameter.

- Insertion of PFN: After confirming satisfactory fracture reduction, an appropriate size nail as determined preoperatively was assembled to insertion handle and inserted manually by slight twisting movements of the hand until the hole for $8 \mathrm{~mm}$ screw was at the level of inferior margin of the neck.

- Insertion of the guide wire for neck screw and hip pin: These were inserted with the help of aiming device lightly screwed to the insertion handle. A $2.7 \mathrm{~mm}$ guide wire was inserted through the drill sleeve. The position of guide wire should be in the lower half of the neck in AP view and in the center of the neck in lateral view. A second $2.7 \mathrm{~mm}$ guide wire was inserted through the drill sleeve above the first one for hip pin. The tip of this guide wire should be approximately $25-20 \mathrm{~mm}$ less deep than planned neck screw.

- Insertion of the neck screw and hip pin: After drilling over the guide wire, the self-tapping $8 \mathrm{~mm}$ neck screw was inserted using cannulated screw driver. Similarly, appropriate length $6.4 \mathrm{~mm}$ hip pin was inserted which was 10-15 mm lesser than neck screw. Length and position of the screws were confirmed with the image intensifier.

- $\quad$ Distal locking: Distal locking was usually performed with two $4.9 \mathrm{~mm}$ distal locking bolts and the position was confirmed with image intensifier.

- $\quad$ Closure: The surgical closure was done in layers and the sterile dressing applied over wound and compression bandage were given. 


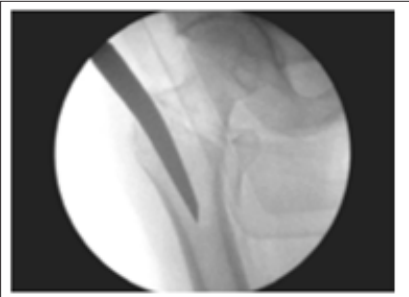

$4 \mathrm{a}$

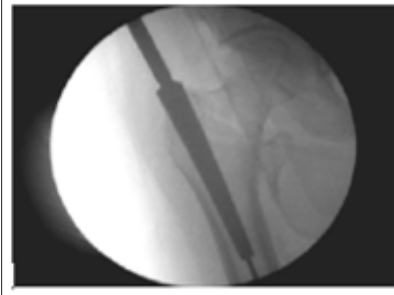

$4 c$

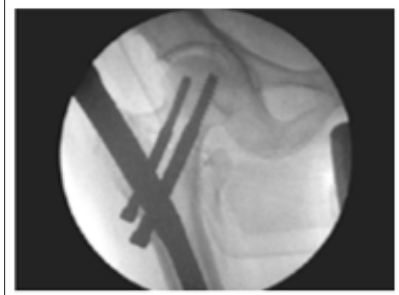

$4 \mathrm{e}$

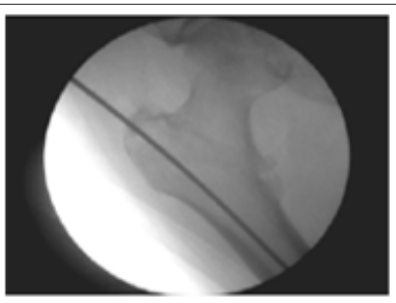

$4 \mathrm{~b}$

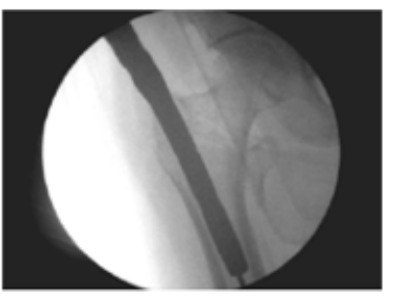

$4 d$

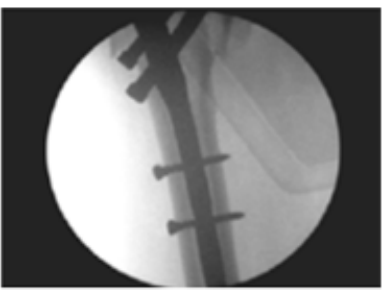

$4 \mathrm{f}$

Figure 4: Surgical technique of PFN insertion - 4a) Nail entry point, 4b) Insertion of guide pin, 4c) Proximal reamer, 4d) Insertion of PFN, 4e) Placement of $6 \mathrm{~mm}$ anti-rotation and $8 \mathrm{~mm}$ compression screws and 4f) Placement of $4.9 \mathrm{~mm}$ distal cortical screws.

Post-operative protocol: Intravenous antibiotics were given for 5 days followed by oral antibiotics for 5 days. Injection Zoledronic acid infusion were administered to improve the degree of osteoporosis. The active and passive exercises were initiated within 48hours of surgery. Partial weight bearing was allowed on $5^{\text {th }}$ postoperative day.

Follow up: Tablet Risedronate 35mg once weekly for 12 weeks were given to improve the bone mineral density in patients with severe degree osteoporosis. All the patients were advised to completely weight bearing after 10-12 weeks of post-operative period. All patients were followed up at the end of 1,2,6,12 and 18 months. At each follow up, the radiographs of upper femur and hip were taken to assess the fracture union and the complications. The functional results were calculated according to Harris hip score. All patients enrolled in the study were offered with surgical implant removal at an average of 18 months post-surgical procedure who showed the clinical and the radiological union of fractures and improved functional quality of life.

\begin{tabular}{||l|l|l|l|}
\hline Pre-op & Immediate post op & $4^{\text {th }}$ month follow up & $15^{\text {th }}$ month follow up \\
\hline \hline & & & \\
\hline
\end{tabular}

Figure 5: Trochanteric fracture fixed with proximal femoral nail. 


\begin{tabular}{||l|l|l|l||}
\hline \hline Pre-op & Immediate post op & $12^{\text {th }}$ month follow up & Implant removal \\
\hline & & & \\
\hline
\end{tabular}

Figure 6: Subtrochanteric fracture fixed with proximal femoral nail.

\section{Results}

A total of 153 cases of proximal femoral fractures [trochanteric $(n=88)$ and subtrochanteric $(n=65)]$ underwent surgical management with proximal femoral nailing as per our study protocol. The descriptive statistics were reported as mean (SD) for continuous variables, frequencies (percentage) for categorical variables. Data were statistically evaluated with IBM SPSS Statistics

Table 4: Patient's demography according to age group. for Windows, Version 20.0, IBM Corp, Chicago, IL.

\section{Demography}

Among 153 cases, 82 cases (53.59\%) were males and 71 cases (46.40\%) were females. All the patients belong to age between 30 to 70 years of age. The maximum age limit in the study was 77 years and minimum age was 32 years. The average age of the patients enrolled in the study were $57.37 \pm 7.12$ years.

\begin{tabular}{|c|c|c|c|c|}
\hline \multirow{2}{*}{ Age group years } & \multicolumn{2}{|c|}{ Group A (n=88) } & \multicolumn{2}{|c|}{ Group B $(n=65)$} \\
\hline & Males & Females & Males & Females \\
\hline $31-40$ & 4 & 1 & 4 & 6 \\
\hline $41-50$ & 15 & 11 & 12 & 8 \\
\hline $51-60$ & 11 & 14 & 7 & 7 \\
\hline $61-70$ & 12 & 9 & 6 & 8 \\
\hline $71-80$ & 7 & 4 & 4 & 3 \\
\hline Total & 49 & 39 & 33 & 32 \\
\hline
\end{tabular}

\section{Mode of injury}

Among 153 cases, 63 cases (41.17\%) sustained injury due to road traffic accident, 38 cases (24.83\%) fall from height and 52 cases (33.98\%) due to trivial fall .

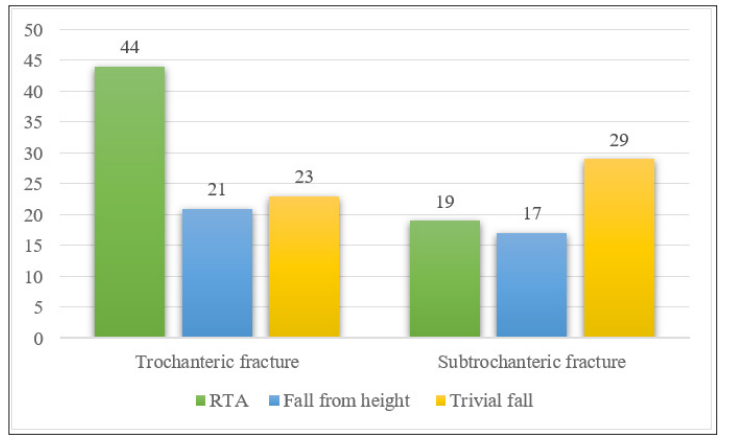

Figure 7: Mode of injury.

\section{Type of fracture pattern}

According to Boyd and Griffin classification, out of 88 cases of trochanteric fracture, the pattern of fractures was type 1 in 5 cases, type 2 in 23 cases, type 3 in 49 cases and type 4 in 11 cases (Figure 8). According to Seinsheimer classification, out of 65 cases of subtrochanteric fracture, the pattern of fractures was type 1 in 5 cases, type 2 in 17 cases, type 3 in 12 cases, type 4 in 29 cases and type 5 in 2 cases.

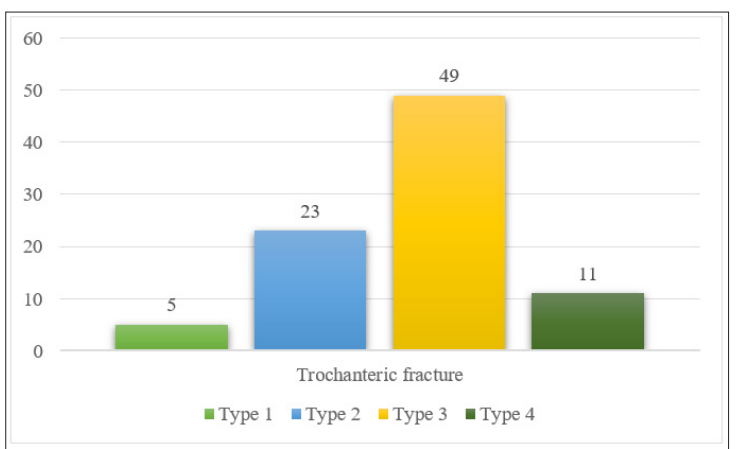

Figure 8: Type of fracture pattern in trochanteric fractures. 


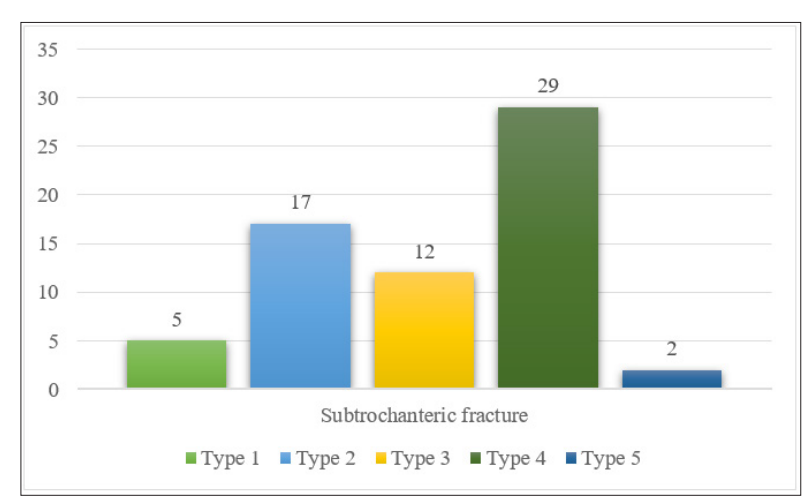

Figure 9: Type of fracture pattern in subtrochanteric fractures.

\section{Closed vs open reduction of fractures}

Among 153 cases, 114 cases (74.50\%) were operated with closed reduction and internal fixation with proximal femoral nailing and 39 cases (25.49\%) underwent open reduction and internal fixation with proximal femoral nailing. The open reduction of fractures was due to delayed presentation for management, inadequate immobilization, interposition of soft tissues and failed closed reduction due to fibrous union.

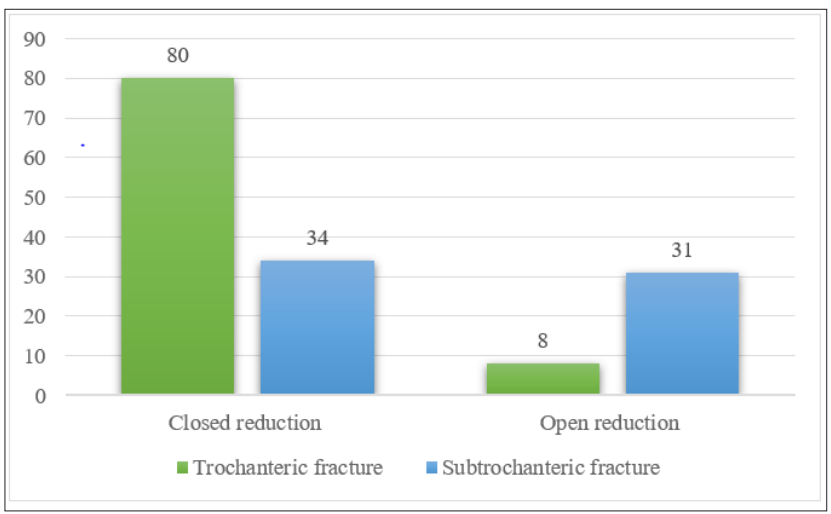

Figure 10: Reduction of fractures.

\section{Details of surgery}

All cases were operated with an average of $2.25 \pm 0.53$ days ranging from 2.10-3.29 days of admission. All cases were treated with closed or open reduction and internal fixation with proximal femoral nailing. The length of the nails was individualized according the diameter of the isthmus. No intraoperative complications were noted during the surgical procedure.

\section{Duration of fracture union}

All the cases were followed up serially with serial clinical and radiographical examinations as per our study protocol. The mean radiological union of trochanteric fractures were $16.73 \pm 2.69$ weeks and subtrochanteric fractures were $19.92 \pm 3.01$ weeks. A total of 2 cases $(1.30 \%)$ of trochanteric fractures and 1 case $(0.65 \%)$ of subtrochanteric fracture showed signs of established non-union after 9 months of post procedure with proximal femoral nailing. A total of 3 cases landed in non-union due to improper immobilization in the post-surgical period.

\section{Complications}

The most common complication encountered in our study was pain 29 cases (18.95\%), delayed union 1 case $(0.65 \%)$, malunion 8 cases (5.22\%), non-union 3 cases (1.96\%), Varus angulation 4 cases (2.61\%), nail breakage 3 cases (1.96\%), screw cut-out 3 cases (1.96\%), Z effect 3 cases (1.96\%), reverse $Z$ effect 2 cases $(1.30 \%)$ and avascular necrosis of femoral head 1 case $(0.65 \%)$. The pain at the fracture site were treated by oral analgesics. Delayed union case, associated with trochanteric fracture presented at 6 months, were treated with dynamization. Non-union cases were counselled for second surgical procedure with dynamic hip screw or hemiarthroplasty in case of intertrochanteric fracture and nail removal with compression plating with bone grafting in case of subtrochanteric fracture. The nail breakage cases were tracked regularly and offered nail removal and re-nailing. The cases with varus angulation got corrected in due course of bony remodelling after the nail removal. Screw cut-out, $\mathrm{Z}$ effect and reverse $\mathrm{Z}$ effect cases were under sentinel surveillance and were offered nail removal when the clinical and radiological signs of established fracture union. AVN of femoral head has been treated with cemented total hip replacement.

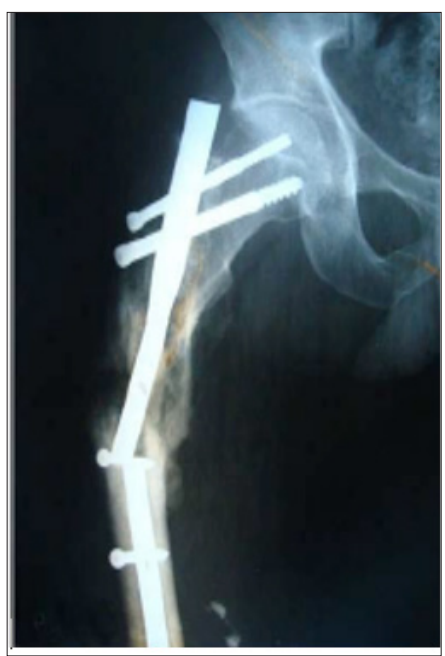

Figure 11a: Nail breakage \& Non-union.

Table 5: Complications.

\begin{tabular}{|c|c|c|}
\hline Complications & $\begin{array}{c}\text { Trochanteric } \\
\text { Fractures }\end{array}$ & $\begin{array}{c}\text { Subtrochan- } \\
\text { teric } \\
\text { Fractures }\end{array}$ \\
\hline Pain & 17 & 12 \\
\hline Delayed union & 1 & - \\
\hline Malunion & 5 & 1 \\
\hline Non-union & 2 & 2 \\
\hline Varus angulation & 2 & 1 \\
\hline Nail breakage & 2 & 1 \\
\hline Screw cut-out & 2 & 1 \\
\hline Z effect & 2 & - \\
\hline Reverse Z effect & 1 & \\
\hline Avascular necrosis of femoral head & 1 & 1 \\
\hline
\end{tabular}




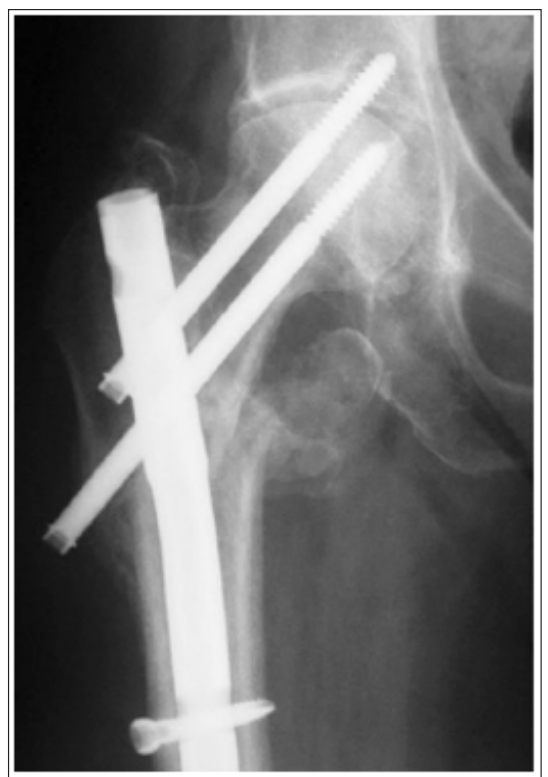

Figure 11b: $Z$ effect.

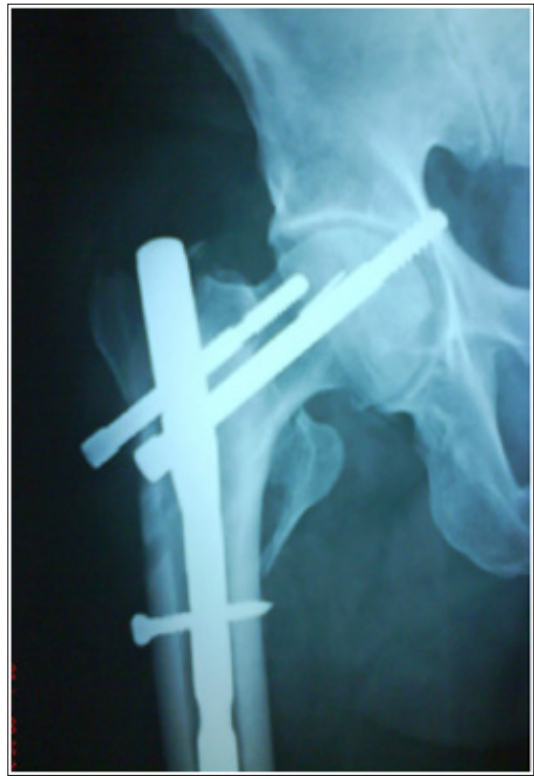

Figure 11c: Reverse $Z$ effect.

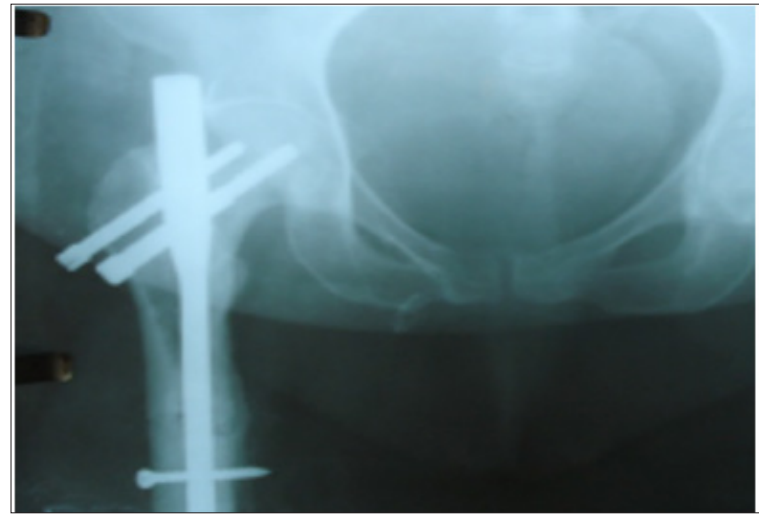

Figure 11d: Screw backout.

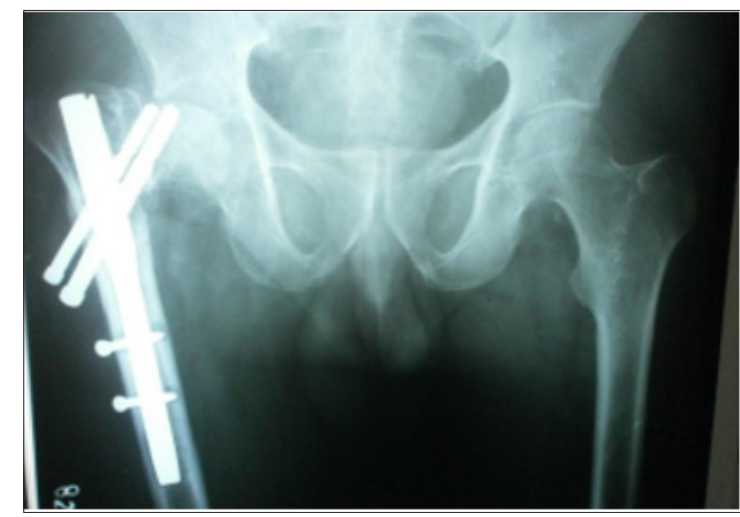

Figure 11e: AVN and cut out of screws at 10 months.

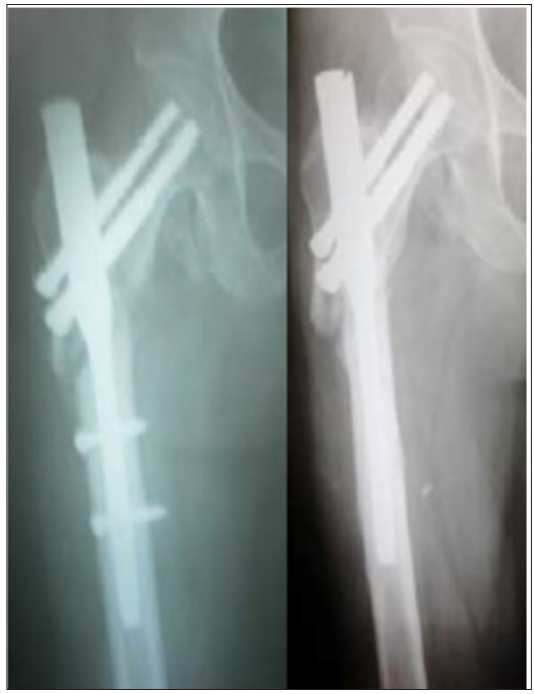

Figure 11f: Delayed union at 6 months and dynamization.

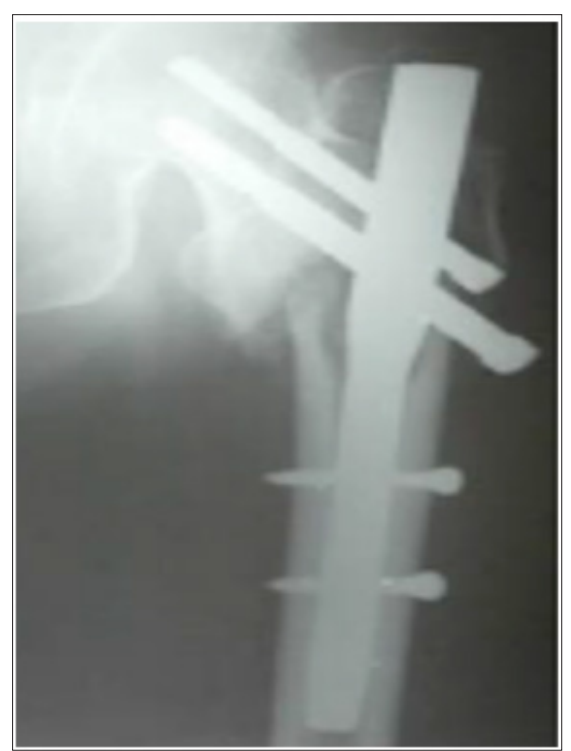

Figure 11g: Compression screw backout at 3 months with Varus collapse. 


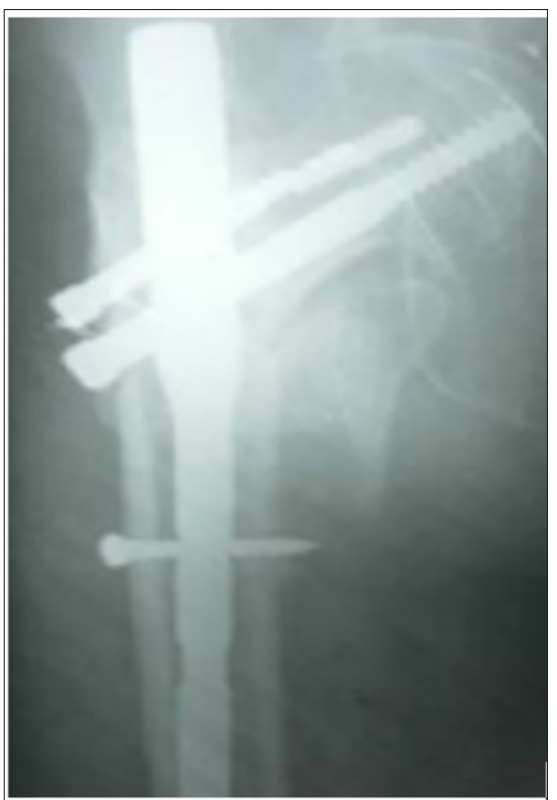

Figure 11h: Varus collapse with anti-rotation screw breakage at 6 months.

\section{Functional assessment with Harris Hip score}

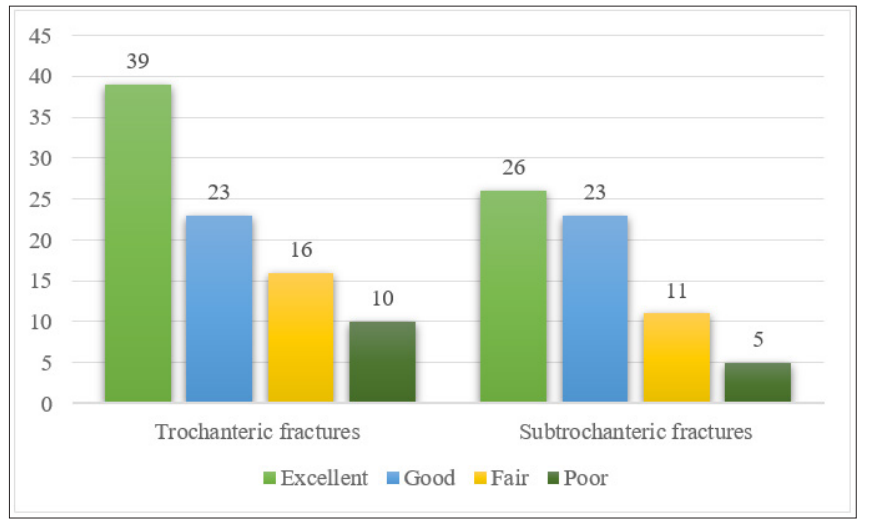

Figure 12: Functional assessment.

All cases were trained with active and passive joint mobilization exercises after clinical sign of fraction union. The functional assessment was made with Harris Hip scores. Out of 153 cases, the functional outcome was excellent in 65 cases (42.48\%), good in 46 cases (30.06\%), fair in 27 cases $(17.64 \%)$ and poor in 15 cases $(9.80 \%)$. The poor range of movements $(n=15)$ were due to nonunion 3 cases, nail breakage 3 cases, screw cut-out 3 cases, Z effect 3 cases, reverse $Z$ effect 2 cases, avascular necrosis of femoral head 1 case.

\section{Surgical implant removal}

Clinically and radiologically united fracture patients were offered with implant removal which were done under loco-regional anaesthesia in an average of $26.32 \pm 3.49$ months.

\section{Discussion}

Proximal femoral fractures represent a significant challenge to the orthopaedic surgeon. These fractures pose a technical difficulty in reduction of fractures and implication of surgical implant may lead to failure of primary fixation of fracture. The medial and posteromedial fracture fragments are the most important elements in determining the severity of trochanteric fracture. In this modern era, researchers stated that the important predictor for re-operation after the trochanteric fracture is the lateral femoral wall [11]. Cephalo-medullary femoral reconstruction nails with a trochanteric entry point are biomechanically stronger than extramedullary implants. In unstable proximal femoral fractures, control of axial telescoping and rotational stability are essential. Intramedullary implants take an advantage of less-invasive procedure which were better tolerated by the elderly and osteoporotic individuals [12]. The various intramedullary devices such as gamma nail, proximal femoral nail, proximal femoral nail anti-rotation, and Intertan integrated nail have been used for fixation of these fractures. The proximal femoral nail compensates for a posteromedial defect acting as a buttress to prevent medialization but fails to provide stability on the lateral side if the lateral wall is compromised. Due to compromise of the lateral wall leads to a situation in which the head of the femur only will resist the medial deforming forces. To prevent Varus collapse, the protection and restoration of abductorlever- arm forces must overcome the deforming forces. Hence restoration of the lateral wall is of paramount importance to prevent varus collapse and further complications [11-13]. In our study, a new cephalo-medullary proximal femoral nail (PFN) for proximal femoral fractures were used. These nails prevent the rotation and collapse of the head-neck fragment and smaller diameter of distal shaft of nail results in less stress concentration at the tip of the nail. The antirotation screw at the proximal aspect of nail increases the biomechanical stability of the fracture fixation.

Prakash et al. [13] studied pro's and cons of PFN in peri trochanteric fractures observed 2 cases of iatrogenic fracture of lateral cortex, 2 cases of failure to put derotation screw and 1 case of guidewire breakage among 20 cases. They concluded that PFN is a reliable implant for peri trochanteric fractures with high rate of bone union and minimal complications. Kashid et al. [14] compared PFN and PFN-A in unstable intertrochanteric fractures in 50 patients and concluded that PFNA significantly reduces the surgical duration, amount of blood loss and fluoroscopic imaging as compared to PFN and no statistical significant benefits over PFN in terms of post-operative functional recovery or complications. Anirudh et al. [15] concluded similar functional outcomes in both PFN and PFN-A implants with fewer complications over PFN-A $(p=0.04)$ in the management of unstable intertrochanteric fractures in 48 patients in osteoporotic settings. Hence, they recommended the use of PFN-A in unstable intertrochanteric fractures with superimposed osteoporosis.

Linga et al. [16] observed the functional outcome of PFN in unstable intertrochanteric fractures in 40 patients and reported that PFN is the implant of choice for unstable IT fractures in terms of fracture union and improvement over pre-injury functional status of the individual. Boblee et al. [17] studied the functional outcome of PFN in 20 patients with trochanteric fractures and concluded that PFN is the ideal implant for the treatment of 
intertrochanteric fractures with negligible varus collapse even in unstable intertrochanteric fractures. Lei et al. [18] concluded long PFN or long gamma nail are the implants of choice in the fixation of subtrochanteric fractures in 49 patients with good bony union at the fracture site with minimal complications [19].

Gadegone WM et al. [19] managed 82 unstable intertrochanteric fractures with PFN concluded that augmentation of PFN with additional screw provides the stability of the construct with minimal complications. Chopra et al. [20] concluded 3 cases of $Z$ effect and 2 cases of reverse $\mathrm{Z}$ effect in a series of 125 cases managed surgically with PFN for unstable intertrochanteric fractures. Werner et al. [21] detected 5 cases of $\mathrm{Z}$ effect (7.1\%) in 70 cases of per and subtrochanteric fractures managed by PFN. They referred Z-effect phenomenon as a characteristic sliding of the proximal antirotation and compression screws to opposite directions during the postoperative weight-bearing period. Boldin et al [22] observed 3 cases of $\mathrm{Z}$ effect and 2 cases of reverse $\mathrm{Z}$ effect in 55 patients of unstable intertrochanteric or subtrochanteric fractures by PFN. They coined reverse Z-effect as an event occurred with movement of the hip pin towards the lateral side, which required early removal.

In our study, we managed both trochanteric $(n=88)$ and subtrochanteric fractures $(n=65)$ surgically by load sharing device named proximal femoral nailing. Among 88 trochanteric fracture cases, 26 cases belong to 41-50 years age group. The most common type of trochanteric fracture operated with PFN was Body and Griffin's type III fracture (Displaced fracture; Fracture line extending to subtrochanteric plane). The mean radiological union of trochanteric fractures were $16.73 \pm 2.69$ weeks. The complications occurred with PFN in trochanteric fracture were pain 17 cases, delayed union 1 case, malunion 5 cases, non-union 2 cases, Varus angulation 2 cases, nail breakage 2 cases, screw cut out 2 cases, $\mathrm{Z}$ effect 2 cases, reverse $\mathrm{Z}$ effect 1 case and avascular necrosis of femoral head 1 case. The functional assessment according to Harris Hip score showed excellent in 39 (44.31\%), good in 23 (26.13\%), fair in $16(18.18 \%)$ and poor in $10(11.36 \%)$ cases.

Among 65 subtrochanteric fracture cases, 20 cases belong to 41-50 years age group. The most common type of subtrochanteric fracture operated with PFN was Seinsheimer's type IV fracture (Comminuted fracture with 4 or more fragments). The mean radiological union of subtrochanteric fractures were $19.92 \pm 3.01$ weeks. The complications occurred with PFN in subtrochanteric fracture were pain 12 cases, malunion 3 cases, non-union 1 case, Varus angulation 2 cases, nail breakage 1 case, screw cut out 1 case, $\mathrm{Z}$ effect 1 case and reverse $\mathrm{Z}$ effect 1 case. The functional assessment according to Harris Hip score showed excellent in 26 (40.00\%), good in 23 (35.38\%), fair in 11 (16.92\%) and poor in $5(7.69 \%)$ cases. Out of 153 cases, the functional outcome was excellent in 65 cases (42.48\%), good in 46 cases (30.06\%), fair in 27 cases $(17.64 \%)$ and poor in 15 cases $(9.80 \%)$. The poor range of movements $(\mathrm{n}=15)$ were due to non-union 3 cases, nail breakage 3 cases, screw cut-out 3 cases, Z effect 3 cases, reverse Z effect 2 cases, avascular necrosis of femoral head 1 case.

Proximal femoral nail has the biological advantages such as closed reduction and less soft tissue dissection, shorter surgical time, least amount of blood loss and early mobilization of patient in the postoperative period and the mechanical advantages such as restoration of abductor-lever-arm mechanism, decreased tensile strain on the implant and maintenance of controlled fracture impaction.

\section{Conclusion}

Proximal femoral fractures pose a great challenge for orthopaedic surgeons to manage surgically. Due to the evolution of load sharing devices with anatomical pre-bent and angulation, it is possible to manage the fractures with internal splinting. We recommend proximal femoral nail as an implant of choice for trochanteric and subtrochanteric fractures of proximal femur which was biomechanically compatible with the stability of the fracture pattern with minimal complications. The load sharing device, proximal femoral nail, decrease the patient related morbidity during intra \& post-operative period and improve the functional status of the patients.

\section{References}

1. Toni MM, Ericka AL (2004) Treatment modalities for subtrochanteric fractures in elderly. Techniques in Orthopaedics 19(3): 197-213.

2. Tyllianakis M, Panagopoulos A, Papadopoulos A, Papasmos S, Mousafiris K (2004) Treatment of extracapsular hip fractures with the proximal femoral nail (PFN): long term results in 45 patients. Acta Orthop Belg 70(5): 444-454.

3. Adams CI, Robinson CM, Court BCM, McQueen MM (2001) Prospective randomized controlled trial of an intramedullary nail versus dynamic screw and plate of intertrochanteric fractures of the femur. J Orthop Trauma 15(6): 394-400.

4. Gupta RK, Sangwan K, Kamboj P, Punia SS, Walecha P (2010) Unstable trochanteric fractures: the role of lateral wall reconstruction. Int Orthop 34(1): 125-129.

5. Chou DT, Taylor AM, Boulton C, Moran CG (2012) Reverse oblique intertrochanteric femoral fractures treated with the intramedullary hip screw (IMHS). Injury 43(6): 817-821.

6. Klinger HM, Baums HM, Eckert M, Neugebauer R (2005) A comparative study of unstable per and intertrochanteric femoral fractures with DHS and PFN and TSP. Zentralbl Chir 130(4): 301-306.

7. Alyassari G, Langstaff RJ, Jones JW, AlLami M (2002) The AO/ASIF proximal femoral nail (PFN) for the treatment of unstable trochanteric femoral fracture. Injury 33(5): 395-399.

8. Gadegone WM, Salphale YS (2007) Proximal femoral nail-an analysis of 100 cases of proximal femoral fractures with an average follow up of 1 year. Int Orthop 31(3): 403-408.

9. Gadegone WM, Salphale YS (2010) Short proximal femoral nail fixation for trochanteric fractures. J Orthop Surg 18(1): 39-44.

10. Pajarinen J, Lindahl J, Michelsson O, Savolainen V, Hirvensalo E (2005) Pertrochanteric femoral fractures treated with a dynamic hip screw or a proximal femoral nail. A randomised study comparing post-operative rehabilitation. J Bone Joint Surg Br 87(1): 76-81.

11. Gotfried Y (2004) The lateral trochanteric wall: a key element in the reconstruction of unstable pertrochanteric hip fractures. Clin Orthop Relat Res 425: 82-86.

12. Palm H, Jacobsen S, Sonne HS, Gebuhr P, Hip Fracture Study Group (2007) Integrity of the lateral femoral wall in intertrochanteric hip fractures: an important predictor of a reoperation. J Bone Joint Surg Am 89(3): 470-475. 
13. Prakash Wali, Santhosh Mared (2018) Pro's and Con's of proximal femoral nail in Peri trochanteric fractures. Int J Orth Sci 4(3): 209-213.

14. Kashid MR, Gogia T, Prabhakara A, Jafri MA, Shaktawat DS, et al. (2016) Comparative study between proximal femoral nail and proximal femoral nail anti-rotation in management of unstable trochanteric fractures. Int J Res Orthop 2(4): 354-358.

15. Anirudh Sharma, Anupam Mahajan, Bobby John (2017) A comparison of the clinico-radiological outcomes with proximal femoral nail (PFN) and proximal femoral nail anti-rotation (PFNA) in fixation of unstable intertrochanteric fractures. J Clin Diagn Res 11(7): RC05-RC09.

16. Linga SS, Aithala JP, Savur A (2017) Can proximal femoral nailing of unstable trochanteric fractures help to regain pre-injury functional status in Indian rural patients? A prospective study of functional outcome of unstable intertrochanteric fractures with proximal femoral nailing. Int J Res Orthop 3(4): 728-33.

17. Boblee James, Ram Prasath, Vijayakumaran (2017) Functional outcome of proximal femoral nailing in intertrochanteric fractures of femur: A prospective study. Int J Orth Sci 3(2): 513-518.
18. Jiang LS, Shen L, Dai LY (2007) Intramedullary fixation of subtrochanteric fractures with long proximal femoral nail or long gamma nail: technical notes and preliminary results. Annals Academy of Medicine 36(10): 821-826.

19. Gadegone WM, Shivashankar B, Lokhande V, Salphale Y (2017) Augmentation of proximal femoral nail in unstable trochanteric fractures. SICOT J 3(12): 1-9.

20. Chopra BL, Kumar K, Khajotia BL, Bhambu R (2017) Proximal femoral nail-outcome and complications: a prospective study of 125 cases of proximal femoral fractures. Int J Res Orthop 3(5): 973-978.

21. Werner TW, Lajtai G, Schmiedhuber G, Lang T, Pirkl C, (2002) Intra and perioperative complications in the stabilization of per and subtrochanteric femoral fractures by means of PFN. Unfallchirurg 105(10): 881885.

22. Boldin C, Seibert FJ, Fankhauser F, Peicha G, Grechenig W, et al. (2003) The proximal femoral nail (PFN) a minimal invasive treatment of unstable proximal femoral fractures: a prospective study of 55 patients with a follow-up of 15 months. Acta Orthop Scand 74(1): 53-58.

For possible submissions Click below: 